\title{
HIGH RESOLUTION COIMPUTED TOMOGRAPHY FINDINGS IN CORONAVIRUS DISEASE 2019 (COVID-19)
}

KEY WORDS: Corona virus, COVID-19, Ground glass opacities, HRCT Chest

\section{Dr Devraj Yadav}

\section{Dr Reena} Mathur*

\section{Dr Rachitha N}

\section{Dr Suresh Jat}

Senior Resident, Department of Radiodiagnosis, JLN Medical College, Ajmer, Raj.

Senior Professor and HOD, Department of Radiodiagnosis, JLN Medical College, Ajmer, Raj*Corresponding Author

Junior Resident, Department of Radiodiagnosis, JLN Medical College, Ajmer, Raj.

Junior Resident, Department of Radiodiagnosis, JLN Medical College, Ajmer, Raj.

Purpose: To assess the HRCT findings of symptomatic COVID-19 patients with positive reverse transcriptase polymerase chain reaction (RT-PCR).Methods: This was a prospective observational study comprising 100 consecutive reverse transcriptase polymerase chain reaction (RT-PCR) positive patients who underwent CT chest. Distribution, extent and type of abnormal lung findings were observed. Results: Among the total study cohort of 100 patients, 64 (64 $\%)$ were males and 36 (36\%) were females with mean age of $42.1 \pm 15.6$ years. We observed lung parenchymalabnormalities in 55(55\%) cases whereas 45 (45\%) RT-PCR positive cases had a normal chest CT. Only $11 \%$ of the patients were dyspneic, $6 \%$ had desaturation, $9 \%$ had increased respiratory rate and $15 \%$ had comorbidities.Among the patients with abnormal CT findings bilateral 44/55 (80\%), multilobar 49/55 (89\%) lung involvement with a predominant peripheral and posterior distribution was commonly observed. With regards to the type ofopacity, ground glass opacity (GGO) was the dominant abnormality found in all 55 (100 \%) cases. Pure GGO was observed in 16 (29.1\%), GGO with septal thickening was seen in 28 (50.9\%) and GGO mixed with consolidation was noted in $11(20 \%)$.Conclusion: In this study population predominantly with mild symptoms and few comorbidities, $45 \%$ ofRT-PCR positive patients had a normal chest CT; whereas the remaining $55 \%$ patients showed typical findings ofpredominant GGOs with a bilateral distribution and peripheral predominance.

\section{INTRODUCTION}

Coronavirus disease 2019 (COVID-19) is a contagious disease caused by severe acute respiratory syndrome coronavirus 2 (SARS-CoV-2). The first case was identified in Wuhan, China, in December 2019. The disease has since spread worldwide, leading to an ongoing pandemic. According to World Health Organization (WHO) more than 6.5 million positive cases have been reported with 387177 deaths as of June $05,2020^{[1]}$.

SARS-CoV-2 is an enveloped single-stranded RNA virus ${ }^{[2,3]}$. The clinical presentation ranges from asymptomatic, mildly symptomatic cases to severely ill ${ }^{[4,5]}$. Typical CT findings include bilateral pulmonary parenchymal ground-glass and consolidative pulmonary opacities, sometimes with a rounded morphology and a peripheral lung distribution. There is a gradual transformation of GGOs into consolidations during the intermediate stage of the disease. The extent of lung abnormalities at CT peaked during illness days 6-1 $1^{[6]}$. Clinical recovery is associated with a gradual resorption of pulmonary opacities with development of subpleural lines, reticulations, fibrous stripes and perilobular opacities, usually apparent after the second week. In some patients the clinical course is complicated by acute respiratory distress syndrome (ARDS) or pulmonary embolism, the main causes of death ${ }^{[7]}$. Pleural effusion, pericardial effusion, mediastinal lymphadenopathy are seen in patients with severe disease ${ }^{[8,9]}$.

\section{MATERIAL AND METHODS}

\subsection{Patient cohort and study design}

This was a prospective observational study from 16, August 2020 to 15, September 2020, conducted in our hospital inJLN Hospital, Ajmer, Rajasthan, India, which was a designated COVID-19 Care Centre (CCC) with separate inpatient, intensive care unit (ICU) and quarantine facilities. 100 symptomatic patients admitted with positive nasopharyngeal swab for SARS-CoV-2 by RT-PCR method were subjected to High resolution CT of chest.

\subsubsection{Inclusion criteria}

Symptomatic patients with RT-PCR confirmed SARS-CoV-2 infection.

\subsubsection{Exclusion criteria}

Asymptomatic patients with RT-PCR confirmed SARS-CoV-2 infection.

Patients with severe illness requiring intensive care and mechanical ventilation were admitted to intensive care unit and those with symptoms but no signs of respiratory failure were admitted in routine wards. Asymptomatic patients were isolated and managed in quarantine facility.

The age, gender, history of contact/travel, clinical symptoms, co-morbid conditions and laboratory investigations of the patients were collected and analysed.

\subsection{CT protocol}

Chest CT was performed between3-7 days after the onset of symptoms. High resolution chest CT was performed using a 16-Slice CT scanner (Philips MX 16) with the following parameters: Tube voltage $90-120 \mathrm{kVp}$, tube current $130-200$ $\mathrm{mAs}$, collimation of $16 \times 0.6$ and a pitch of 0.75 .The CT images were acquired in a single inspiratory breath-hold.Images were reconstructed using increment of $0.5 \mathrm{~mm}$ to $1 \mathrm{~mm}$ thick slices.The images were viewed in both lung window settings (width 1200-1500 HU; centering -500 to $-700 \mathrm{HU}$ ) and mediastinal window (width $300400 \mathrm{HU}$; centering 40HU). After every scan decontamination of the CT suite was performed using $0.1 \%$ sodium hypochlorite.

\subsection{CT Image interpretation}

The CT patterns were described according to the terms defined by the Fleischner Society and peer-reviewed literature on viral pneumonia ${ }^{(10,1)}$. The CT images were assessed for the presence of ground-glass opacity (hazy areas of increased attenuation without obscuration of the underlying vasculature), consolidation (homogeneous 
opacification with obscuration of the underlying vasculature), reticular pattern (consisting of either coarse linear or curvilinear opacity or fine subpleural reticulation without substantial ground-glass opacity) and mixed pattern (combination of consolidation, ground-glass opacity, and reticular opacity in the presence of architectural distortion). Perilesional and intralesional vasculature were assessed for vascular dilatation. Furthermore, the involvement was described based on (a)axial distribution- central (central $2 / 3^{\text {rd }}$ )or peripheral (peripheral $1 / 3^{\text {rd }}$ ) or diffuse involvement, (b) involvement of one or both lung and(c)lobar involvement. In addition, the presence of pleura effusion, pneumothorax, emphysema, or mediastinal lymphadenopathy (short axis diameter $>1.0 \mathrm{~cm}$ ) were also noted.

\section{RESULTS}

3.1. Demographics, clinical characteristics and laboratory findings

Out of a cohort of 100 patients included in the study, 64 (64\%) were males and $36(36 \%)$ were females with mean age of $42.1 \pm 15.6$ years (range 20-74 years). A history of close contact with an infected patient or a history of travel to a highrisk zone within or outside the country was present in $68 \%$ of cases. Fever was the commonest symptom seen in $55(55 \%)$ followed by fatigue or malaise in $43(43 \%)$, cough in 35(35\%) and sore throat in $32(32 \%)$. Only $11(11 \%)$ of the patients were dyspnoeic, $9(9 \%)$ had increased respiratory rate and 6 $(6 \%)$ had desaturation. Anosmia and loss of taste were seen in $8(8 \%)$, diarrhoea in $3(3 \%)$ and abdominal pain in $2(2 \%)$ cases.Comorbidities were present in $15(15 \%)$ patients. Creactive protein was elevated in $59(59 \%)$ patients.Patient demographics, clinical features and laboratory investigations are summarized in Table 1.

Table 1 Demographics, Clinical features, comorbidities and Laboratory investigations in COVID-19 positive patients.

\begin{tabular}{|c|c|}
\hline Patient demographics & $\begin{array}{c}\text { Number of patients (\%) } \\
(\mathrm{n}=100)\end{array}$ \\
\hline Mean age(years) \pm S.D & $\begin{array}{c}42.1 \pm 15.6 \\
\text { years }\end{array}$ \\
\hline \multicolumn{2}{|l|}{ Gender } \\
\hline Male & 64 \\
\hline Female & 36 \\
\hline \multicolumn{2}{|l|}{$\begin{array}{c}\text { History of contact with a COVID- } \\
19 \text { patient o travel to a high risk } \\
\text { zone } \\
\end{array}$} \\
\hline Present & 62 \\
\hline Absent & 38 \\
\hline Co-morbid illness & 15 \\
\hline Hypertension & 10 \\
\hline Diabetes Mellitus & 9 \\
\hline Bronchial asthma & 2 \\
\hline Chronic obstructive lungdisease & 2 \\
\hline \multicolumn{2}{|l|}{ Clinical features } \\
\hline Fever & 55 \\
\hline Cough & 35 \\
\hline Sore throat & 32 \\
\hline Malaise/Fatigue & 43 \\
\hline Anosmia & 8 \\
\hline Loss of taste & 8 \\
\hline Diarrhea & 3 \\
\hline Abdominal pain & 2 \\
\hline Dyspnea & 11 \\
\hline \begin{tabular}{|c} 
Increased Respiratory rate $(>30$ \\
$/ \mathrm{min})$
\end{tabular} & 9 \\
\hline $\begin{array}{c}\text { Reduced Oxygen Saturation }(< \\
90 \%)\end{array}$ & 6 \\
\hline
\end{tabular}

\begin{tabular}{|c|c|}
\hline Lab Investigations & \\
\hline $\begin{array}{c}\text { Increased C-Reactive protein } \\
\text { (normal value }<10 \mathrm{mg} / \mathrm{L})\end{array}$ & 59 \\
\hline
\end{tabular}

\subsection{Chest CT findings}

Lung parenchymal abnormalities were observed in 55 (55\%) cases, whereas 45 (45\%) RT-PCR positive cases had a normal chest CT. Among the patients with abnormal CT findings, bilateral lung involvement was the commonest, observed in $44(80 \%)$. Multiple lobe involvement was seen more frequently $49(89.1 \%) .25$ (45.4\%)had involvement of all the 5 lobes whereas two lobe and single lobe involvement was seen in $7(12.7 \%)$ and $6(10.9 \%)$ respectively. In terms of axial distribution, peripheral distribution was the commonest, seen in $55(100 \%)$ cases among which $43(78.2 \%)$ had only peripheral distribution whereas as $12(21.8 \%)$ had both peripheral and central distribution. None of the patients showed purely central distribution.

\begin{tabular}{|c|c|c|}
\hline Findings & $\begin{array}{c}\text { No. of Patients }(\mathrm{n}= \\
100)\end{array}$ & $\%$ \\
\hline \multicolumn{3}{|l|}{ 1. Findings } \\
\hline Present & 55 & 55 \\
\hline Absent & 45 & 45 \\
\hline \multicolumn{3}{|l|}{ 2. Opacities } \\
\hline Pure GGO & 16 & 29.1 \\
\hline GGO with septal thickening & 28 & 50.9 \\
\hline Pure consolidation & 0 & 0 \\
\hline GGO withConsolidation & 11 & 20 \\
\hline \multicolumn{3}{|l|}{ 3. Lung involvement } \\
\hline Unilateral & 11 & 20 \\
\hline \begin{tabular}{|l|} 
Bilateral \\
\end{tabular} & 44 & 80 \\
\hline \multicolumn{3}{|l|}{ 4. Axial Distribution } \\
\hline Central & 0 & 0 \\
\hline Peripheral & 43 & 78.2 \\
\hline \begin{tabular}{|l|} 
Diffuse \\
\end{tabular} & 12 & 21.8 \\
\hline \multicolumn{3}{|l|}{ 5. Vascular involvement } \\
\hline Present & 8 & 14.5 \\
\hline Absent & 47 & 85.5 \\
\hline \multicolumn{3}{|l|}{ 6. Other findings } \\
\hline Pleural Effusion & 3 & 5.5 \\
\hline Pneumothorax & 0 & 0 \\
\hline Mediastinal Lymphadenopathy & 5 & 9.1 \\
\hline Emphysema & 4 & 7.3 \\
\hline Fibrosis & 2 & 3.6 \\
\hline \begin{tabular}{|l|} 
Lung parenchymal \\
Abnormalities \\
\end{tabular} & Number of patient & $\%$ \\
\hline \multicolumn{3}{|l|}{ Lobar Involvement } \\
\hline Right upper & 32 & 58 \\
\hline Right Middle & 28 & 50.1 \\
\hline \begin{tabular}{|l} 
Right Lower \\
\end{tabular} & 31 & 56.4 \\
\hline Left Upper & 33 & 60 \\
\hline Left Lower & 26 & 47.3 \\
\hline \multicolumn{3}{|l|}{ No of Lobes } \\
\hline 5 & 25 & 45.4 \\
\hline 4 & 13 & 23.6 \\
\hline 3 & 4 & 7.3 \\
\hline 2 & 7 & 12.7 \\
\hline 1 & 6 & 10.9 \\
\hline \multicolumn{3}{|l|}{ CT Severity Index } \\
\hline $1-8$ & 36 & 65.5 \\
\hline $9-15$ & 11 & 20 \\
\hline $16-25$ & 8 & 14.5 \\
\hline
\end{tabular}




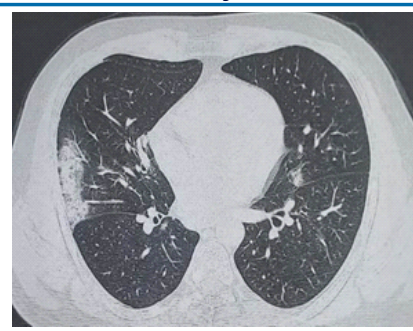

Fig (a)

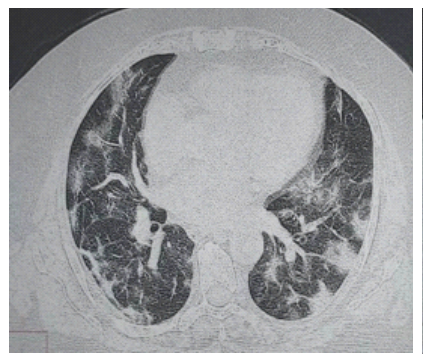

Fig (c)

Non-contrast axial chest CT images inthe lung window setting of a 48-year-old maleCOVID-19 positive patient, obtained 7 daysafter symptom onset, at the carinal (a), upper lobes (b), mid-basal (c) and basal (d) levelsshowing bilateral elongated, confluent groundglass opacities with pronounced peripheral andposterior distribution with interlobular septalthickening producing crazy-paving patternwith early progression to consolidation formation.

\section{DISSCUSSION}

Various studies have been done on radiological findings of COVID-19 pneumonia. Many asymptomatic patients can have a positive chest HRCT findingwhereas it has also been observed that many symptomatic patients can have a negative CT especially during the early phase ofthe illness ${ }^{[12]}$. A prospective observational study was conducted at JLN Hospital, Ajmer, Rajasthanto describe the HRCTfindings of symptomatic COVID-19 patients in our population. There were $65.5 \%$ mild, $20 \%$ moderate and $14.5 \%$ severe-critical illness cases in thisstudy. In an environmentally homogenous cohort (Diamond PrincessCruise ship), Inui S et.al ${ }^{[12]}$ reported a normal chest CT in $21 \%$ ofsymptomatic COVID-19 cases with cough (20\%), fever (11\%) anddyspnoea (3\%). They further observed that nearly half $(54 \%)$ of thesymptomatic cases had an abnormal CT. In contrast, $55 \%$ of the cases in our study with varying severity of symptoms had abnormal CT.The low prevalence of CTfindings in laboratory confirmed symptomatic SARS-CoV-2 patients in our population compared to the reported data from other countries raises the possibility of divergentcourse of the disease in different populations. Putatively,three factors inisolation or in varying combinations could account for this discrepancy.First, a low prevalence of abnormal CT scans in our population maybe because CT scans were performed in very early phase of the disease SARS-CoV-2, withmost patients having a mild illness.Second, a low prevalence of comorbidities in our study cohort withno known immune compromised patients (like cancer patients onchemotherapy) may have contributed to low CT positivity rate. Co-morbid illnesses are known to be associated with increased severity of COVID-19 disease ${ }^{[13]}$. Third, it may be reflective of a less severe form of the diseasein our population which is tentatively indicated by low CFR in ourpopulation so far. The less severity of the disease may in turn resultfrom a less virulent strain of virus or a robust immune status of the population. The loss of follow-up imaging precludes us from conclusivelyrefuting this possibility. Weimaged patients between 3-7 days after thesymptom onset.Among the patients with lung parenchymal abnormalities on HRCT, bilateral and multilobar distribution of pulmonary opacities with aperipheral distribution was commonly observed. Our results fairlycorroborate the distribution and type of pulmonary opacities reportedin COVID-19 pneumonia. The most common lung parenchymal abnormalities encountered were GGO in the form of pure GGO (29.1\%), GGO with septal thickening $(50.9 \%)$ or GGO admixed with consolidation (20\%). The various putactiveetiologiesthat have been put forth to account for this uniquefinding ofvascularenlargement include,vasodilatation induced by the release ofproinflammatory cytokines, small vessel pulmonary embolism and infection induced pulmonary vasculitis ${ }^{[14]}$ Thefinding of pulmonary avascular enlargement seems to have a diagnostic value as it has notbeen reported previously in any infectious disease settings. Bai et al. ${ }^{[15]}$ reported vascular enlargement to be frequently associated withCOVID-19 pneumonia compared to non-COVID-19 pneumonia. The presence of enlarged vessel sign mayhelp discriminate COVID-19 pneumonia from non-COVID-19 pneumonia. However, the small number of patientswith severe disease precluded us from performing a meaningful correlation analysis within the subsets.CT is not recommended as a screening tool for the diagnosis ofCOVID$19^{[16,17]}$. However, CT can contribute to the clinical management of the COVID-19 disease and in our understanding of thedisease. According to the German Radiological Society, CT may aid inassessing the initial extent of the lung involvement, help in recognitionof the pneumoniaassociated complications and also help in monitoringthe progression of the disease in severe cases ${ }^{[16]}$. According toAmerican College of Radiology (ACR) guidelines, CT should be reservedfor hospitalized, symptomatic patients with specific clinical indicationslike worsening respiratory status ${ }^{[17]}$ There are several limitations to our study. First, we focused on initial or baseline CTfindings and did not perform follow-up CT examinations. This may result in non-inclusion of symptomatic cases thatmay have developed lung changes late in the course of disease andhence a spurious high rate of negative CTs in the study population.Second, there may have been a selection bias as imaging was performedin all symptomatic cases regardless of the severity of illness. The smallsize of study population is also a limitation.

The CT findings of COVID-19 pneumonia reflected a typical lung injury of viral pneumonia, which was characterized by a rapid change as seen in severe acute respiratory syndrome and Middle East respiratory syndrome ${ }^{[11,18,19]}$

\section{REFERENCES:}

1. https://www.who.int/emergencies/diseases/novel-coronavirus-2019

2. Y. Chen, L. Li, SARS-CoV-2: virus dynamics and host response, Lancet Infect. Dis.(March 2020)

3. C. Huang, Y. Wang, X. Li, et al., Clinical features of patients infected with 2019 novel coronavirus in Wuhan, China, Lancet 395 (10223) (2020) 497-506, https://doi.org/10.1016/s0140-6736(20)30183-5.

4. W.H. Kong, Y. Li, M.W. Peng, D.G. Kong, X.B.Yang, L.Wang, M.Q. Liu, SARS-CoV2detection in patients with influenza-like illness, Nat. Microbiol. (April) (2020) 1-4.

5. Y. Yang, M. Yang, C. Shen, et al., Evaluating the Accuracy of Different RespiratorySpecimens in the Laboratory Diagnosis and Monitoring the Viral Shedding of 2019-nCoV Infections, (2020), https://doi.org/10.1101/ 2020.02.11.20021493.

6. Temporal Changes of CT Findings in 90 Patients with COVID-19 Pneumonia: A Longitudinal Study,https://pubs.rsna.org/doi/full/10.1148/radiol. 2020200843

7. A.H. Parry, A.H. Wani, Pulmonary embolism in coronavirus disease-19 (COVID-19)and use of compression ultrasonography in its optimal management,Thromb. Res. (May 2020).

8. M. Chung, A. Bernheim, X. Mei, et al., CT imaging features of 2019 novel coronavirus (2019-nCoV), Radiology (2020) 200230,https://doi.org/ 10.1148/radiol.2020200230.

9. A. Bernheim, X. Mei, M. Huang, et al., Chest CT findings in coronavirus Disease-19(COVID-19):relationship to duration of infection, Radiology (2020) 200463 https://doi.org/10.1148/radiol.2020200463.

10. Hansell DM, Bankier AA, MacMahon H, McLoud TC, Müller NL, Remy J. Fleischner Society: glossary of terms for thoracic imaging. Radiology 2008;246(3):697-722.

11. Ooi GC, Khong PL, Müller NL, et al. Severe acute respiratory syndrome: temporallung changes at thin-section $\mathrm{CT}$ in 30 patients. Radiology 2004;230(3):836-844.]

12. S. Inui, A. Fujikawa, M. Jitsu, N. Kunishima, S. Watanabe, Y. Suzuki, S. Umeda,Y.Uwabe, Chest CTfindings in cases from the cruise ship"DiamondPrincess"withcoronavirus disease 2019 (COVID-19), 
Radiology 2 (March (2)) (2020) e200110.

13. F. Zhou, T.Yu, R. Du, G. Fan, Y. Liu, Z. Liu, J. Xiang,Y.Wang, B. Song, X. Gu,L. Guan,

Clinical course and risk factors for mortality of adult inpatients withCOVID-

19 in Wuhan, China: a retrospective cohort study, Lancet (March) (2020).

14. A.H.Parry, A.H. Wani, Segmental pulmonary vascular changes in COVID19 pneumonia, Am. J. Roentgenol. (May) (2020)Wl-

15. H.X. Bai, B. Hsieh, Z. Xiong, K. Halsey, J.W. Choi, T.M. Tran, I. Pan, L.B. Shi,D.C. Wang, J. Mei, X.L. Jiang, Performance of radiologists in differentiating COVID19 from viral pneumonia on chest CT, Radiology (March) (2020) 200823

16. J. Vogel-Claussen, J. Ley-Zaporozhan, P. Agarwal, J. Biederer, H.U. Kauczor, S. Ley,H.Kühl, U.G. Mueller-Lisse, T. Persigehl, C.L. Schlett, D. Wormanns, Recommendations of the Thoracic Imaging Section of the German RadiologicalSociety for Clinical Application of Chest Imaging and Structured CT Reporting inthe COVID-19 Pandemic, InRöFo-Fortschritte auf demGebiet der Röntgenstrahlenund der bildgebendenVerfahren, (c) Georg Thieme Verlag KG,2020 May 26.

17. https://www.acr.org/Advocacy-and-Economics/ACR-PositionStatements/Recommendations-for-Chest-Radiography-and-CT-forSuspected-COVID19-Infection. Accessed 25/05/2020-

18. Antonio GE, Ooi CG, Wong KT, et al. Radiographic-clinical correlation in severe acute respiratory syndrome: study of 1373 patients in Hong Kong. Radiology 2005;237(3): 1081-1090.

19. Das KM, Lee EY, Enani MA, et al. CT correlation with outcomes in 15 patients with acute Middle East respiratory syndrome coronavirus. AJR Am J Roentgenol 2015;204(4):736-742 